\title{
Study of Astrophysical s-Process Neutron Capture Reactions at the High- Intensity SARAF-LiLiT Neutron Source
}

\author{
Michael Paul ${ }^{1, *}$, Moshe Tessler ${ }^{2}$, Shlomi Halfon ${ }^{2}$, Elad Korngut ${ }^{1}$, Arik Kreisel ${ }^{2}$, Tala Palchan ${ }^{1}$, Eliran Peretz ${ }^{1}$, \\ Leonid Weissman ${ }^{2}$, and Asher Shor ${ }^{2}$ \\ ${ }^{1}$ Racah Institute of Physics, Hebrew University, Jerusalem, Israel 91904 \\ ${ }^{2}$ Soreq Nuclear Research Center, Yavneh, Israel, 81800
}

\begin{abstract}
We report on recent experiments at the Soreq Applied Research Accelerator Facility - Liquid-Lithium Target (SARAF-LiLiT) laboratory dedicated to the study of $s$-process neutron capture reactions. The kW-power proton beam at $1.92 \mathrm{MeV}$ (1-2 mA) from SARAF Phase I yields high-intensity $30 \mathrm{keV}$ quasi-Maxwellian neutrons $\left(3-5 \times 10^{10} \mathrm{n} / \mathrm{s}\right)$. The high neutron intensity enables Maxwellian averaged cross sections (MACS) measurements of low-abundance or radioactive targets. Neutron capture reactions on the important $s$-process branching points ${ }^{147} \mathrm{Pm}$ and ${ }^{171} \mathrm{Tm}$ were investigated by activation in the LiLiT neutron beam and $\gamma$-measurements of their decay products. MACS values at $30 \mathrm{keV}$ extracted from the experimental spectrum-averaged cross sections are obtained and will be discussed. The Kr region, at the border between the so-called weak and strong $s$-process was also investigated. Atom Trap Trace Analysis (ATTA) was used for the first time for the measurement of a nuclear reaction cross section. After activation in the quasi-Maxwellian neutron flux at SARAF-LiLiT, isotopic ratios were determined for ${ }^{81} \mathrm{Kr}(230 \mathrm{ky}) /{ }^{80} \mathrm{Kr}$ and ${ }^{85 g} \mathrm{Kr}(10.8 \mathrm{y}) /{ }^{84} \mathrm{Kr}$. The latter ratio was confirmed both by low-level $\beta$ counting and $\gamma$ spectrometry. The shorter-lived capture products ${ }^{79,85 m, 87} \mathrm{Kr}$ were detected by $\gamma$-spectrometry and the corresponding neutron-capture MACS of the respective target nuclei ${ }^{78,84,86} \mathrm{Kr}$ were determined. The MACS of the ${ }^{80} \mathrm{Kr}(\mathrm{n}, \gamma){ }^{81} \mathrm{Kr}$ and ${ }^{84} \mathrm{Kr}(\mathrm{n}, \gamma){ }^{85 g} \mathrm{Kr}$ reactions are still under study. The partial MACS leading to ${ }^{85 m} \operatorname{Kr}(4.5 \mathrm{~h})$ measured in this experiment has interesting implications since this state decays preferentially by $\gamma$ decay (79\%) to ${ }^{85} \mathrm{Rb}$ on a faster time scale than does ${ }^{859} \mathrm{Kr}$ and behaves thus as an $s$-process branching point.
\end{abstract}

\section{Introduction}

Except for the lightest among them $(\mathrm{H}, \mathrm{He}, \mathrm{Li})$ that were created during the nucleosynthesis era of the Big Bang, the nuclei of the elements are produced in stars. Neutrons generated in-situ, and in particular their captures on nuclear seeds, are responsible for the production of the large majority of the heavier nuclides $(\mathrm{A} \geq 60)$ [1, 2].

In this article we address mainly, on an experimental basis, one of the neutron-induced nucleosynthesis paths, namely the so-called slow neutron capture process $(s-$ process) [3, 4]. The $s$-process consists of a succession of neutron captures, each occurring over a mean time longer than or commensurate with the typical mean life of $\beta^{-}$decaying nuclides close to the valley of stability. The $s$-process is believed to occur in stars during their $\mathrm{He}$ core and shell burning stages, where neutrons are available from $(\alpha, n)$ reactions. In contrast, the rapid neutron capture process ( $r$-process) consists of the sequential captures of a large number $(\approx 20)$ of neutrons on a fast time scale, much shorter than $\beta$-decaying times, occurring in an astrophysical site with an extreme neutron density $\left(\approx 10^{20}-10^{22}\right.$ $\left.\mathrm{cm}^{-3}\right)[3,4]$.

*e-mail: paul@vms.huji.ac.il
We review here an experimental program conducted at the Soreq Applied Research Accelerator Facility (SARAF) centered on the study of neutron-capture reactions along the astrophysical $s$-process path. The long-standing interest in the study of such reactions and the availability of a high-intensity proton beam $(\approx 2 \mathrm{~mA})$ at SARAF (Phase I) led us to develop a new type of intense acceleratorbased neutron source based on the ${ }^{7} \operatorname{Li}(p, n)^{7} \mathrm{Be}$ reaction near threshold using for the first time a Liquid-Lithium Target (LiLiT) capable of dissipating a continuous beam power up to $\approx 10 \mathrm{~kW}$ for neutron production. This development increased the neutron intensity available from the ${ }^{7} \mathrm{Li}(p, n)$ reaction $\left(3-5 \times 10^{10} \mathrm{n} / \mathrm{s}\right)$ by a factor of $30-50$ compared to existing facilities.

\section{The Soreq Applied Research Accelerator Facility (SARAF) and the Liquid-Lithium Target (LiLiT): activation experiments}

The SARAF accelerator [5, 6] is a superconducting linear RF accelerator designed to produce a beam of light ions $(m / q \leq 2)$ with a continuous-wave $(\mathrm{CW})$ beam intensity up to $5 \mathrm{~mA}$. The layout of Phase $\mathrm{I}$ is shown in Fig. 1. Light-ion $\mathrm{H}^{+}$or $\mathrm{D}^{+}$are extracted at $20 \mathrm{keV} / u$ from 
an Electron Cyclotron Resonance (ECR) ion source, massanalyzed and injected via a Low-Energy Beam Transport (LEBT) line to a $176 \mathrm{MHz}$ four-rod Radio-FrequencyQuadrupole (RFQ) with a final energy of $1.5 \mathrm{MeV} / u$. A short Medium-Energy Beam (MEBT) line transports the beam to a Prototype Superconducting Module (PSM) housing six Half-Wave Resonators (HWR) followed by the High Energy Beam (HEBT) line towards the LiLiT target at Station 1 (Fig. 1 via two $45^{\circ}$ bending magnets.

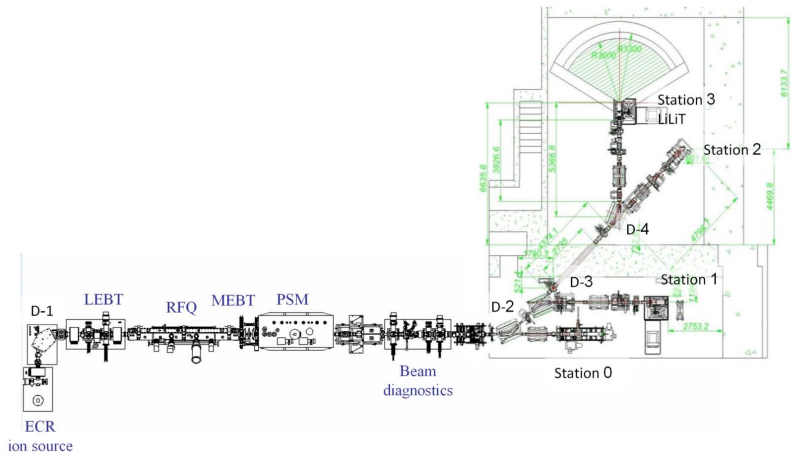

Figure 1. Layout of SARAF Phase I. The recent extension to a new target room (Phase I+), is shown in green; see text for acronyms. The experiments described in Section 3 were performed at Station 1.

We describe here briefly the principle, the technical design and major components of the Liquid-Lithium Target (LiLiT) [7]. The target was specifically designed and built to dissipate the high power of the $\mathrm{CW}$ proton beam from SARAF (up to $\approx 5 \mathrm{~kW}$ ) and produce quasi-Maxwellian neutrons for nuclear astrophysics experiments. Liquid lithium is forced-flown from the top into a $15 \mathrm{~mm}$ wide, $1.8 \mathrm{~mm}$ thick film supported by a thin stainless steel wall (concave as viewed in the proton beam direction). The proton beam impinges directly (windowless) onto the lithium surface emitting neutrons mainly in the forward direction. The liquid lithium acts both as a (thick) target producing neutrons near the surface $(\approx 4 \mu \mathrm{m}$ deep $)$ and as a beam dump. The high power $\left(\approx 0.7 \mathrm{MW} / \mathrm{cm}^{3}\right)$ at the Bragg peak depth is mechanically transported by the fast flow $(2-5 \mathrm{~m} / \mathrm{s})$ to a reservoir and heat exchanger. The lithium surface temperature at the position of the beam impingement has been studied experimentally with an infrared (IR) thermal camera to verify the model estimations [8]. Photographs of the lithium surface taken with an optical and IR camera during irradiation are shown in Fig. 2

The complex mechanical structure of the LiLiT setup prevents any reliable direct measurement of the neutron intensity and energy distribution as seen by the secondary target. While the Au monitor foils (see below) provide a useful measure of the integrated neutron flux, their interpretation and the eventual extraction of a quantitative cross section require knowledge of the neutron energy and angular distributions. An extensive effort was therefore dedicated to the development and benchmarking of detailed simulations for the analysis of activation experiments. The

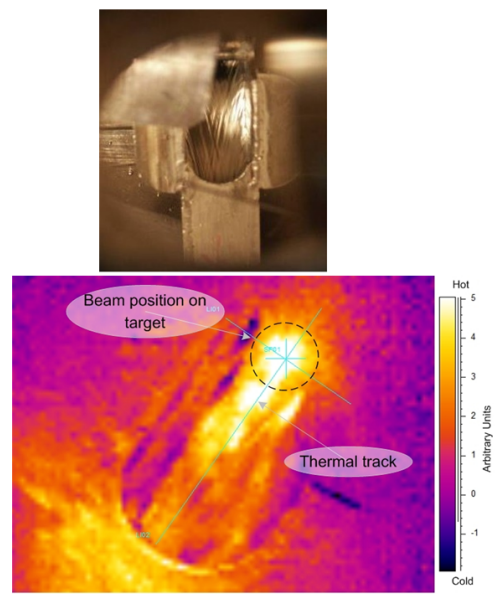

Figure 2. (top) Photographs of the liquid-lithium flow taken with an optical camera; (bottom) a beam-on beam-off subtracted IR image of the lithium flow. The figure is adapted from [8].

simulation code we developed is divided in two parts the first of which, named SimLiT [9], calculates the neutron field produced in given conditions by LiLiT. The neutrons are then transported using the code GEANT4 [10] taking into account the geometry of the secondary target and surrounding components and materials (Fig. 3) .

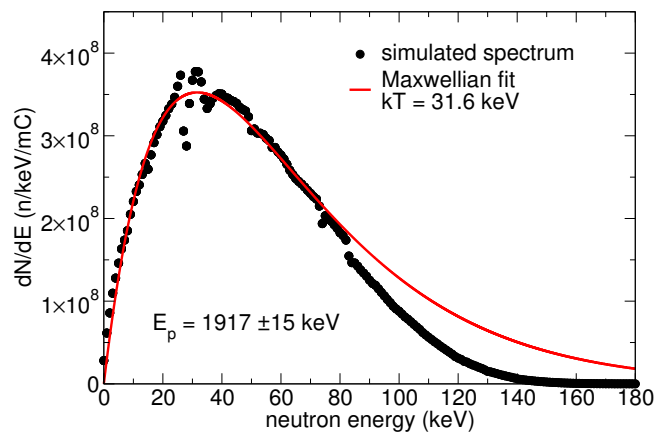

Figure 3. A simulated neutron spectrum impinging on the $\mathrm{Zr}$ target for the Exp II irradiation. Figure adapted from [11].

An activation measurement consists of irradiation of a secondary target in the neutron field generated by the ${ }^{7} \mathrm{Li}(p, n)$ reaction in LiLiT. An experimental cross section for the neutron-induced reaction is extracted from the offline decay counting of the activated target or by atom counting of the residual nuclide. The target must be positioned as close as possible to the Li film to maximize the neutron dose. The experimental setup is illustrated in Fig. 4. The secondary target is located in a so-called experimental chamber separated from the LiLiT chamber by a curved vacuum wall. The proton beam (full width of $\approx 9 \mathrm{~mm}$ ) impinges on the free surface of the liquid lithium film, resulting in outgoing forward-directed neutrons. The wall curvature allows the secondary target within its holder to be at a distance of $6 \pm 1 \mathrm{~mm}$ from the liquid-Li surface. Standard foil targets are $25 \mathrm{~mm}$ diameter but larger foils up to $40 \mathrm{~mm}$ diameter can be mounted in the holder; the secondary target is typically sandwiched within the holder 
between two Au foils serving as neutron monitors. During an irradiation run, the experimental chamber is evacuated (rough vacuum of $\approx 10^{-3}$ mbar) and serves also as a safety buffer in case of a LiLiT failure causing the intense proton beam to pierce through the separation wall. A shaft (Fig. 4), inserted through an end port to the experimental chamber, is used to load and secure the target holder in position by a bayonet clamp and is removed from the chamber before irradiation. The setup and simulations were care-

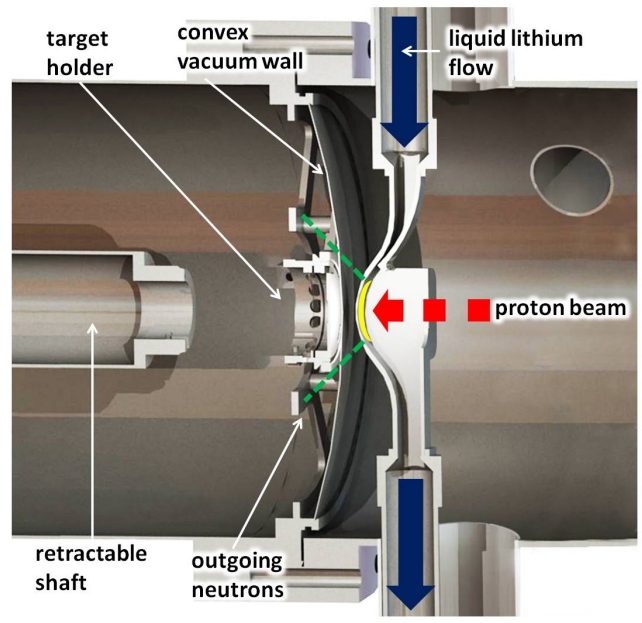

Figure 4. Diagram of the Liquid-Lithium Target (LiLiT) and activation target assembly. The (1-2 $\mathrm{mA}, \approx 9 \mathrm{~mm}$ full width) proton beam (dashed red arrow) impinges on the free-surface lithium film (yellow crescent). The solid blue arrows show the inlet and outlet of the external circulating loop (see $|7|$ for details). The activation targets are mounted on a circular holder and positioned in the outgoing neutron cone (green dotted lines) at a distance of $\approx 6 \mathrm{~mm}$ from the lithium surface in a vacuum chamber separated from the LiLiT chamber by a $0.5 \mathrm{~mm}$ stainless steel wall convex to the beam. The retractable shaft (at left) is used to load and unload rapidly the target assembly. The shaft is removed before the irradiation. Adapted from [11].

fully benchmarked in [11] through measurements of the ${ }^{94,96} \mathrm{Zr}(\mathrm{n}, \gamma)^{95,97} \mathrm{Zr}$ cross sections where a method of extrapolation of experimental cross sections to Maxwellianaveraged cross sections (MACS) is described.

\section{3 (n, $\gamma$ ) experiments completed and in progress at SARAF-LiLiT}

We describe here experiments, recently published or in progress, where the detection of the neutron-capture products is performed by decay counting via $\gamma$ spectrometry or by atom counting methods.

\subsection{The ${ }^{147} \operatorname{Pm}(\mathrm{n}, \gamma){ }^{148 g, m} \mathrm{Pm}$ reactions}

The high-intensity neutron beam of the SARAF-LiLiT neutron source enables us to measure neutron capture cross sections of target of very small mass and especially radioactive targets. We report here the recent determination of the ${ }^{147} \mathrm{Pm}(n, \gamma){ }^{148 g, m} \mathrm{Pm}$ cross sections [12], performed on a $56 \mu \mathrm{g}$ high-purity ${ }^{147} \mathrm{Pm}$ target. The target was made by neutron irradiation of a ${ }^{146} \mathrm{Nd}_{2} \mathrm{O}_{3}$ pellet, enriched to $98.8 \%$ in ${ }^{146} \mathrm{Nd}$, for 54 days with a thermal flux of $10^{15} \mathrm{n} / \mathrm{cm}^{2} / \mathrm{s}$ at ILL, followed, after radioactive cooling, by $\mathrm{Pm}$ chemical separation at PSI [13]. Figure 5 shows the activation set-up and includes details on the target (deposited on a $5 \mu \mathrm{m}, 27 \mathrm{~mm}$ diameter $\mathrm{Al}$ foil and mounted between two mylar foils in a $80 \mathrm{~mm}$ diameter fiberglass epoxy resin ring) placed in an $\mathrm{Al}$ holder at $6 \mathrm{~mm}$ from the lithium target surface. LiLiT was bombarded during $\approx 4$

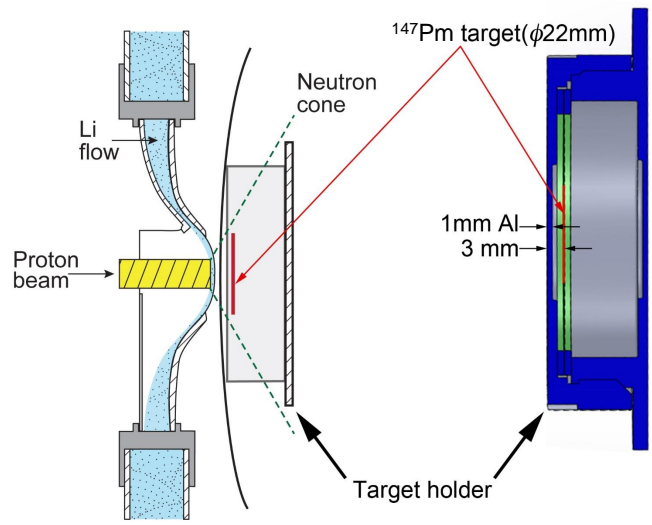

Figure 5. Schematic diagram of the activation setup: the ${ }^{147} \mathrm{Pm}$ target in an airtight $\mathrm{Al}$ holder is located just downstream of the curved wall (thick solid line) separating the vacuum of the LiLiT chamber and that of the activation chamber.

hours by a $\approx 1.5 \mathrm{~mA}$ proton beam at $1930 \mathrm{keV}$ resulting in a quasi-Maxwellian fluence of $\approx 1.2 \times 10^{14}$ neutrons $/ \mathrm{cm}^{2}$. $\mathrm{Au}$ monitors were placed on both sides of the Al target holder. The activated ${ }^{147} \mathrm{Pm}$ target was counted with a calibrated HPGe gamma spectrometer (Fig. 6) and activation yields of ${ }^{148 g, m} \mathrm{Pm}$ were determined. An absorber setup made of $2 \mathrm{~mm}$ of $\mathrm{Cu}$ and $6 \mathrm{~mm}$ of $\mathrm{Pb}$ was installed between the target and the detector in order to minimize the background from low energy photons from the target and from bremsstrahlung related to the $\beta$-decay. A careful determination of the photopeak efficiency of the Ge detector was made by placing calibration sources in a dummy Al holder with the same absorber setup. Detailed simulations made with the SimLiT [9] and GEANT4 [10] codes were performed to obtain the energy distributions of neutrons incident on the ${ }^{147} \mathrm{Pm}$ target and ${ }^{197} \mathrm{Au}$ monitor. Experimental ${ }^{147} \mathrm{Pm}(n, \gamma){ }^{148 g, m} \mathrm{Pm}$ cross sections were determined relative to the spectrum-averaged ${ }^{197} \mathrm{Au}(\mathrm{n}, \gamma){ }^{198} \mathrm{Au}$ cross section, using the evaluated point-wise cross sections JEFF-3.3 [14]. A correction factor calculated as described in [11] is applied to extrapolate the experimental cross sections to Maxwellian-averaged cross sections. Table 1 summarizes our results and compares them with those reported by Reifarth et al. [15].

\subsection{The ${ }^{36,38} \operatorname{Ar}(\mathrm{n}, \gamma)^{37,39} \mathrm{Ar}$ reactions}

Accelerator mass spectrometry (AMS, see [17] for a general recent review of the technique) is an ultra-sensitive method of atom counting used mainly for long-lived radioactive nuclides. The technique is applicable for the 


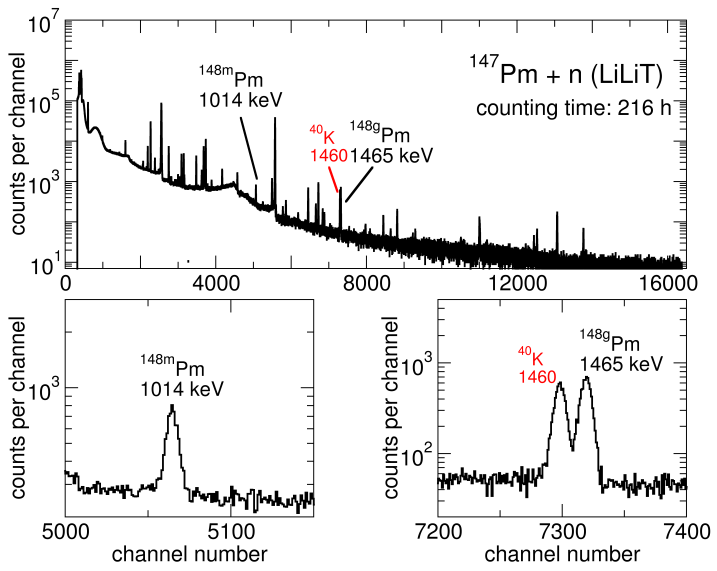

Figure 6. (top) $\gamma$-ray spectrum of the ${ }^{147} \mathrm{Pm}$ target after activation recorded at a distance of $55 \mathrm{~mm}$ from the HPGe detector with the absorber configuration described in the text; (bottom) zoom in regions of the 1013.8 and $1465.12 \mathrm{keV}$ photopeaks from the decay of ${ }^{148 m} \mathrm{Pm}$ and ${ }^{148 g} \mathrm{Pm}$, respectively.

Table 1. Summary of the experimental values for the partial and total MACS at $30 \mathrm{keV}$ for the $\left.{ }^{147} \mathrm{Pm}(n, \gamma)\right)$ reaction.

\begin{tabular}{lccc}
\hline \hline & ${ }^{147 \rightarrow 148 g} \mathrm{Pm}$ & ${ }^{147 \rightarrow 148 m} \mathrm{Pm}$ & Ratio $(\mathrm{g} / \mathrm{m})$ \\
\hline This work & $469(50) \mathrm{mb}$ & $357(27) \mathrm{mb}$ & 1.31 \\
Reifarth $^{1}$ & $332(64) \mathrm{mb}$ & $419(58) \mathrm{mb}$ & 0.79 \\
Difference & $+41 \%$ & $-15 \%$ & \\
& & & \\
\hline
\end{tabular}

${ }^{1}$ Original values scaled up $6 \%$ because of new ${ }^{197} \mathrm{Au}$ standard [16].

detection of reaction products for which decay counting is not practical due either to a very long half-life or decay radiation difficult to detect. We recently used the AMS atom-counting method for the study of the ${ }^{38} \operatorname{Ar}(n, \gamma)$ ${ }^{39} \operatorname{Ar}\left(269\right.$ y) and ${ }^{36} \operatorname{Ar}(n, \gamma){ }^{37} \operatorname{Ar}(35.0 \mathrm{~d})$ reactions after neutron irradiation at SARAF-LiLiT [18]. The ${ }^{36} \mathrm{Ar}$ and ${ }^{38} \mathrm{Ar}$ argon isotopes are among the rare stable nuclides for which the neutron-capture cross sections above thermal energy was never measured experimentally. Although the ${ }^{36,38} \mathrm{Ar}$ abundances in terrestrial atmospheric argon are very small relative to ${ }^{40} \mathrm{Ar}$ (produced mainly from ${ }^{40} \mathrm{~K}$ decay [19, 20] $),{ }^{36} \mathrm{Ar}(84.59 \%)$ and ${ }^{38} \mathrm{Ar}(15.38 \%)$ are the major argon isotopes in the solar system [21] and likely so in stellar matter. They are expected, together with the branching point ${ }^{39} \mathrm{Ar}$, to play a significant role in nucleosynthesis of light neutron-rich nuclei $\left(e . g .{ }^{36} \mathrm{~S},{ }^{40} \mathrm{Ar}\right.$, ${ }^{40} \mathrm{~K}$ ), believed to be produced during the weak $s$-process phase of stellar evolution [22, 23].

Enriched ${ }^{36} \mathrm{Ar}(99.935 \%)$ or ${ }^{38} \mathrm{Ar}(99.957 \%)$ gas samples were filled by compression and cryogenic pumping into separate $10 \mathrm{~mm}$ diameter Ti spheres [24] and irradiated at SARAF-LiLiT. Each sphere was irradiated with a Au foil for neutron fluence monitoring. We performed the AMS experiments for measurement of the ${ }^{39} \mathrm{Ar} /{ }^{38} \mathrm{Ar}$ and ${ }^{37} \mathrm{Ar} /{ }^{36} \mathrm{Ar}$ ratios at the ATLAS facility of Argonne National Laboratory (ANL). Unlike conventional AMS facilities based on electrostatic accelerators and negative ion injection (not suitable for noble gases), ATLAS generates highly-charged positive ions $\left({ }^{A} \mathrm{Ar}^{8+}\right)$ in an ECR ion source, accelerated by a superconducting linac to an energy of $\approx 6 \mathrm{MeV} / u$ (Fig. 7). ${ }^{37,38,39,40} \mathrm{Ar}^{8+}$ ions were al-

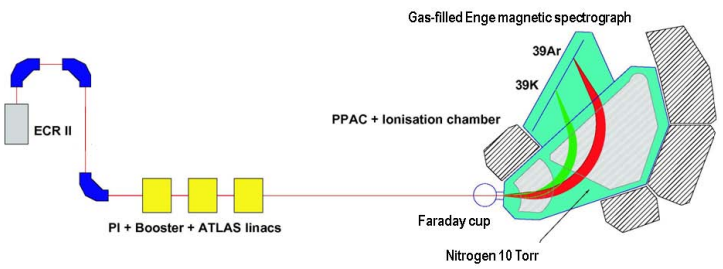

Figure 7. Schematic diagram of the AMS setup at ANL. The ${ }^{39} \mathrm{Ar}^{8+}$ ions from the ECR II ion source are accelerated through the ATLAS accelerator and then analyzed by the Enge gas-filled magnetic spectrograph [25], which physically separates them from beam contaminants with close-by $\mathrm{m} / \mathrm{q}$ values.

ternately accelerated: ${ }^{39} \mathrm{Ar}^{8+}$ ions were analyzed in the Enge gas-filled magnetic spectrograph [25], which physically separated ${ }^{39} \mathrm{Ar}$ from beam contaminants with closeby $\mathrm{m} / \mathrm{q}$ values, e.g. ${ }^{39} \mathrm{~K}^{8+}$ and ${ }^{34} \mathrm{~S}^{7+}$ (Fig. 8). ${ }^{38} \mathrm{Ar}^{8+}$ and ${ }^{40} \mathrm{Ar}^{8+}$ beam intensities were measured in a suppressed Faraday cup in front of the magnetic spectrograph. The

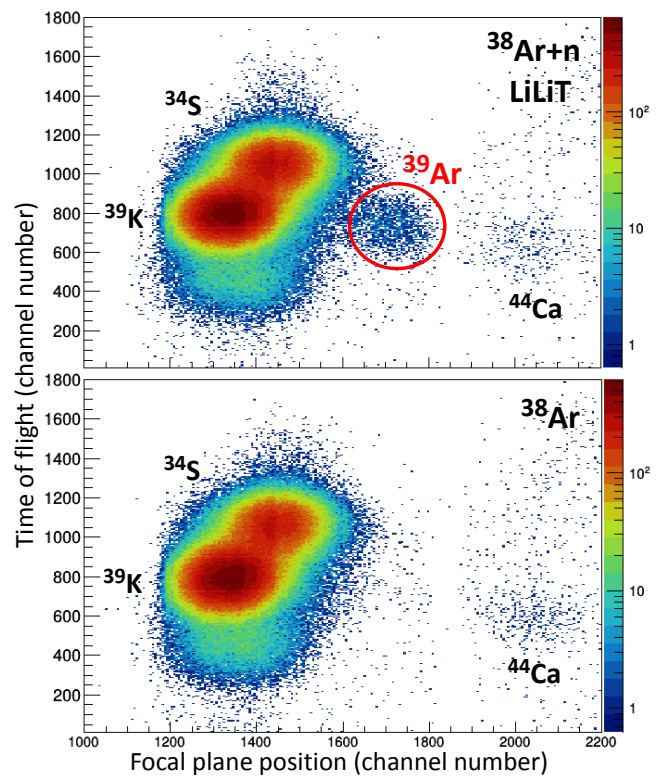

Figure 8. AMS identification spectrum of ${ }^{39} \mathrm{Ar}$ ions in the detector measured for the LiLiT irradiated ${ }^{38} \mathrm{Ar}$ gas (top) and for non-irradiated ${ }^{38} \mathrm{Ar}$ gas (bottom). The horizontal axis represents dispersion along the focal plane and the vertical axis the time-offlight.

${ }^{37} \mathrm{Ar}$ nuclide, decaying by pure electron capture with no $\gamma$-ray emission, is conventionally detected by Auger electron counting, as notoriously done in R. Davis' solar neutrino experiment [26]. We detected and counted ${ }^{37} \mathrm{Ar}$ for the first time by AMS, measuring the ${ }^{37} \mathrm{Ar} /{ }^{36} \mathrm{Ar}$ ratio of 
the irradiated samples. Ar gas was fed from the sphere container into the ECR ion source. ${ }^{36,37} \mathrm{Ar}^{8+}$ ions, extracted from the ion source, were accelerated alternately through ATLAS at an energy of $6 \mathrm{MeV} / u$. It was found necessary to strip (using a $200 \mu \mathrm{g} / \mathrm{cm}^{2} \mathrm{C}$ foil) the ${ }^{37} \mathrm{Ar}^{8+}$ ions and count ${ }^{37} \mathrm{Ar}^{18+}$ (fully stripped) in order to suppress the strong ${ }^{37} \mathrm{Cl}(\mathrm{Z}=17)$ background. The ${ }^{37} \mathrm{Ar}^{18+}$ ions were counted using a $\Delta \mathrm{E}$-E telescope of Si detectors. The ${ }^{36} \mathrm{Ar}^{18+}$ beam intensity was measured in a suppressed Faraday cup located in front of the Si detectors. The ${ }^{37} \mathrm{Ar}$ activity, measured by Auger electrons, was also determined in aliquots of the same gas samples by low-level counting (LLC) in an underground laboratory [27, 28] at the University of Bern. Excellent agreement was obtained between the two methods [18].

The isotopic ratios $r={ }^{A+1} \mathrm{Ar} /{ }^{A} \mathrm{Ar}$ at the end of irradiation are determined by

$$
r=\frac{N_{A+1}}{\epsilon t} \frac{q e}{10^{-9} i_{A}} e^{\lambda t_{c o o l}},
$$

where $N_{A+1}$ is the number of ${ }^{A+1} \mathrm{Ar}$ detected, $\epsilon$ is the detector efficiency, $t$ the counting time, $q$ the ion charge state (18 for ${ }^{37} \mathrm{Ar}$ and 8 for ${ }^{39} \mathrm{Ar}$ ), $e$ the electronic charge in $\mathrm{C}$, and $i_{A}$ the ${ }^{A} \mathrm{Ar}^{q+}$ beam intensity (nA); $\lambda=\frac{\ln (2)}{t_{1 / 2}}$ is the ${ }^{A+1} \mathrm{Ar}$ decay constant and $t_{\text {cool }}$ is the time between end of irradiation and counting. The ${ }^{A+1} \mathrm{Ar} /{ }^{A} \mathrm{Ar}$ ratios measured lead to spectrum-averaged capture cross sections of $\sigma_{\text {exp }}=1.4(1)$ and $0.95(10) \mathrm{mb}$ for ${ }^{36} \mathrm{Ar}$ and ${ }^{38} \mathrm{Ar}$ (see [18]) in the conditions of our experiments. The MACS at a given thermal energy $k T$ were extracted from these experimental cross sections with the procedure developed in [11]) as above, using Hauser-Feshbach models (e.g. [29]) for the energy dependence of the ${ }^{36,38} \operatorname{Ar}(n, \gamma)$ cross sections. Comparisons of our MACS at $30 \mathrm{keV}$ with theoretical and evaluated values for ${ }^{36} \mathrm{Ar}$ and ${ }^{38} \mathrm{Ar}$ are illustrated in Fig. 9. showing notable differences.

Calculations done using the single-zone NucNet Tools reaction network code [30] starting at the H-burning phase with solar abundances [21] and continuing into a singlezone He core burning $\left(\mathrm{T}=300 \mathrm{MK}\right.$, density of $1 \mathrm{~kg} / \mathrm{cm}^{3}$ ) show significant (10-50\%) changes [18] in the calculated mass fractions for neutron-rich light nuclides between ${ }^{34} \mathrm{~S}$ and ${ }^{58} \mathrm{Fe}$, reminiscent of the sensitivity observed in the weak $s$-process region $(\mathrm{A} \approx 56-70)$ due to the change of a single cross section [31].

\subsection{Activation measurements of ${ }^{\text {nat }} \mathrm{Kr}$ and ATTA detection of the ${ }^{81} \mathrm{Kr}$ neutron-capture product}

The neutron capture cross sections of the $\mathrm{Kr}$ isotopes are of special interest for $s$-process nucleosynthesis; the krypton isotopes ${ }^{80,82} \mathrm{Kr}$ for example are solely produced by $s$-process nucleosynthesis due to the fact that they are shielded from the $r$-process by ${ }^{80,82} \mathrm{Se}$. The branching at ${ }^{85} \mathrm{Kr}[32]$ is especially important due to the $\operatorname{direct} \beta$-decay of a ${ }^{85 m} \mathrm{Kr} 4.5 \mathrm{~h}$ isomeric state to ${ }^{85} \mathrm{Rb}$. The cross section for the reaction populating directly the isomeric state, and hence to ${ }^{85} \mathrm{Rb}$, will serve as a tool to estimate the stellar neutron density.

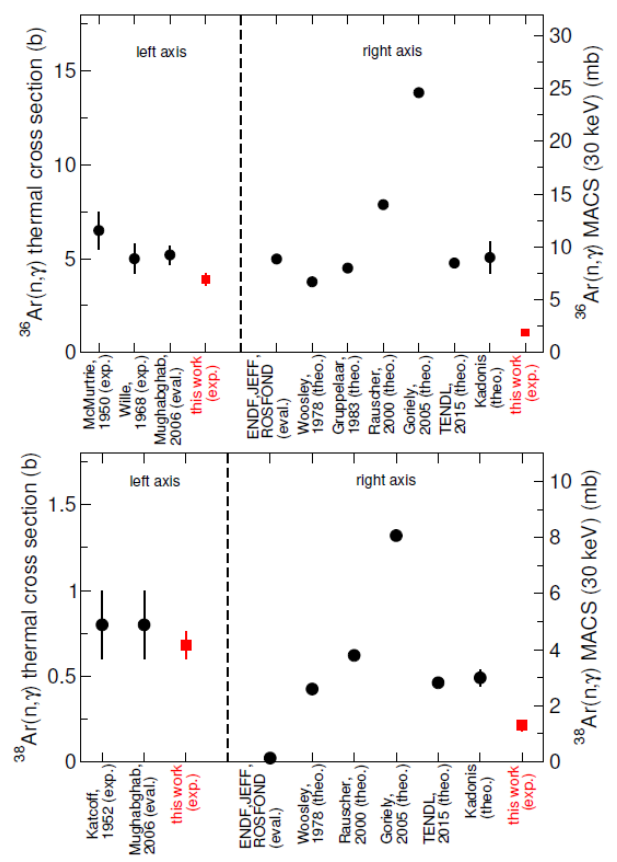

Figure 9. (right panels) Comparison of ${ }^{36,36} \mathrm{Ar}$ neutron capture $30 \mathrm{keV}$ MACS's measured in this work with theoretical values and evaluations. Interpretation of the observed substantial discrepancies are being studied; (left panels) thermal cross sections measured in this work for ${ }^{36,36} \mathrm{Ar}$ are compared to previous experimental results and show good agreement.

Here we report on recent on activation experiments done at SARAF-LiLiT by combining decay counting by $\gamma$ spectroscopy for short-lived products with another atom counting method, namely Atom Trap Trace Analysis (ATTA, [33]). A Ti sphere with $107.7 \mathrm{mg}$ of ${ }^{\text {nat }} \mathrm{Kr}$ was irradiated at SARAF-LiLiT at $\mathrm{E}_{p}=1930 \mathrm{keV}$ for $7.28 \mathrm{~mA} \mathrm{~h}$. The activities of the short-lived $\mathrm{Kr}$ isotopes $\left({ }^{79,87} \mathrm{Kr}\right)$ as well as ${ }^{85 m} \mathrm{Kr}(10.8 \mathrm{y})$ were measured with a High-Purity $\mathrm{Ge}$ (HPGe) detector.

The long-lived $\mathrm{Kr}$ isotopes $\left({ }^{81} \mathrm{Kr}\left(\mathrm{t}_{1 / 2}=2.3 \times 10^{5} \mathrm{y}\right)\right.$ and ${ }^{85} \mathrm{Kr}(10.8 \mathrm{y})$ were measured by Atom Trap Trace Analysis (ATTA) at the ATTA-3 laboratory at ANL [34]: an atom of a particular isotope is selectively captured and repeatedly excited by resonant laser light in a magneto-optical trap (MOT) and detected by observing its fluorescence. The isotopic ratios are measured by trapping successively the rare isotope and a reference isotope by shifting the laser frequency accordingly and comparing the measured ratios to those of a $\mathrm{Kr}$ sample considered as standard. Results of these experiments are under analysis and will be submitted for publication.

\section{Acknowledgments}

We are pleased to thank collaborators from U. of Seville, Paul Scherrer Institute, Institut Laue-Langevin, Argonne National Laboratory, Clemson University, University of Bern, University of Science and Technology of China, Goethe University of Frankfurt and University of Notre Dame in experiments reported in this article. We would 
like to thank the SARAF and LiLiT (Soreq NRC) and the ATLAS (Argonne National Laboratory) operation staffs for their dedicated help during the experiments. This work was supported in part by Pazy Foundation (Israel). M.P. gratefully acknowledges the support of Israel Science Foundation (Grant Nr. 1387/15). We gratefully acknowledge the support of Israel Ministry of Science and Technology. M.T. gratefully acknowledges the support of the Eshkol Foundation of Israel Ministry of Science and Technology.

\section{References}

[1] A. G. W. Cameron, Stellar Evolution, Nuclear Astrophysics, and Nucleogenesis (Dover Publications Inc., 2013), ISBN: 9780486498553.

[2] E. M. Burbidge, G. R. Burbidge, W. A. Fowler, F. Hoyle, Rev. Mod. Phys. 29, 547 (1957).

[3] F. Käppeler, Prog. Part. Nucl. Phys. 43, 419 (1999).

[4] F. Käppeler, R. Gallino, S. Bisterzo, W. Aoki, Rev. Mod. Phys. 83, 157 (2011).

[5] A. Kreisel, L. Weissman, A. Arenshtam, et al. in Proceedings of Linac 2014, Geneva, Switzerland, WEIOB02, (2014).

[6] I. Mardor, O. Aviv, M. Avrigeanu, et al., Eur. Phys. J. A 54, 91 (2018).

[7] M. Paul, M. Tessler, M. Friedman, et al., Eur. Phys. J. A 55, 44 (2019).

[8] L. Danon, Masters thesis, unpublished, Ben Gurion University of the Negev, Beer Sheva, Israel, (2017).

[9] M. Friedman, D. Cohen, M. Paul, et al., Nucl. Instrum. Meth. A 698, 117 (2013).

[10] S. Agostinelli, J. Allison, K. Amako, et al., Nucl. Instrum. Meth. A 506, 250 (2003).

[11] M. Tessler, M. Paul, A. Arenshtam, et al., Phys. Lett. B 751, 418 (2015).

[12] C. Guerrero, M. Tessler, M. Paul, et al., Phys. Lett. B 797, 134809 (2019).

[13] S. Heinitz, E. Maugeri, D. Schumann, et al., Radiochim. Acta 105, 801 (2017).
[14] JEFF collaboration, Joint Evaluated Fission and Fusion File (JEFF) (2017), https://www.oecd-nea.org/ dbdata/jeff/jeff33/.

[15] R. Reifarth, C. Arlandini, M. Heil, et al., Astrophys. J. 582, 1251 (2003).

[16] A. Carlson, V. Pronyaev, R. Capote, et al., Nucl. Data Sheets 148, 143 (2018).

[17] W. Kutschera, Adv. Phys. X 1, 570 (2016).

[18] M. Tessler, M. Paul, S. Halfon, et al., Phys. Rev. Lett. 121, 112701 (2018).

[19] C. F. von Weizsaeker, Phys. Z. 38, 623 (1937).

[20] E. Anders, T. Owen, Science 198, 453 (1977).

[21] K. Lodders, Astrophys. J. 591, 1220 (2003).

[22] R. D. Hoffman, S. E. Woosley, T. A. Weaver, et al., Astrophys. J. 521, 735 (1999)

[23] R. Reifarth, K. Schwarz, F. Käppeler, Astrophys. J. 528, 573 (2000).

[24] G. Rupp, D. Petrich, F. Käppeler, et al., Nucl. Instrum. Meth. A 608, 152 (2009).

[25] M. Paul, B. G. Glagola, W. Henning, et al., Nucl. Instrum. Meth. A 277, 418 (1989).

[26] R. Davis, Prog. Part. Nucl. Phys. 32, 13 (1994).

[27] H. Loosli, M. Möll, H. Oeschger, U. Schotterer, Nucl. Instrum. Meth. B 17, 402 (1986).

[28] R. A. Riedmann, R. Purtschert, Environ. Sci. Technol. 45, 8656 (2011).

[29] S. H. A. Koning, S. Goriely, TALYS-1.8, A Nuclear Reaction Program, NRG-1755 ZG Petten, The Netherlands (2015). http://www.talys.eu/home/.

[30] https://sourceforge.net/projects/nucnet-tools/.

[31] H. Nassar, M. Paul, I. Ahmad, et al., Phys. Rev. Lett. 94, 092504 (2005).

[32] H. Beer, R. L. Macklin, Astrophys. J. 339, 962 (1989).

[33] C. Chen, Y. Li, K. Bailey, T. O’Connor, L. Young, Z.T. Lu, Science 286, 1139 (1999).

[34] W. Jiang, K. Bailey, Z. T. Lu, et al., Geochim. Cosmochim. Ac. 91, 1 (2012). 